2

3 4

5

Short report eLife

\title{
Gain of channel function and modified gating properties in TRPM3 mutants causing
} intellectual disability and epilepsy

\author{
Evelien Van Hoeymissen ${ }^{1,2, \sim}$, Katharina Held ${ }^{1,2, \sim}$, Ana Cristina Nogueira Freitas ${ }^{2, \sim}$, Annelies \\ Janssens $^{2}$, Thomas Voets ${ }^{2, *}, \#$ and Joris Vriens ${ }^{1, *}, \#$
}

\section{Author affiliations:}

${ }^{1}$ Laboratory of Endometrium, Endometriosis and Reproductive Medicine, Department of Development and Regeneration, KU Leuven, Belgium

${ }^{2}$ Laboratory of Ion Channel Research, VIB-KU Leuven Center for Brain and Disease Research, Leuven, Belgium and Department of Molecular Medicine, KU Leuven, Belgium

Shared first authors

* Shared last and corresponding authors

\section{\# Corresponding authors:}

Prof. Joris Vriens

Laboratory of Endometrium, Endometriosis and Reproductive Medicine

Department of Development and Regeneration, KU Leuven

Herestraat 49 box 611, 3000 Leuven, Belgium

Phone: +32 16327279

Email: Joris.Vriens@kuleuven.be

Prof. Thomas Voets

Laboratory of ion channel Research

VIB-KU Leuven Center for Brain and Disease Research and KU Leuven Department of

Molecular Medicine

Herestraat 49 box 801, 3000 Leuven, Belgium

Phone: +32163

Email: Thomas.Voets@kuleuven.VIB.be 


\section{Abstract}

Developmental and epileptic encephalopathies (DEE) are a heterogeneous group of disorders characterized by epilepsy with comorbid intellectual disability. Recently, two de novo heterozygous mutations in the gene encoding TRPM3, a calcium permeable ion channel, were identified as the cause of DEE in eight probands, but the functional consequences of the mutations remained elusive. Here we demonstrate that both mutations (V990M and P1090Q) have distinct effects on TRPM3 gating, including increased basal activity, higher sensitivity to stimulation by the endogenous neurosteroid pregnenolone sulphate (PS) and heat, and altered response to ligand modulation. Most strikingly, the V990M mutation affected the gating of the non-canonical pore of TRPM3, resulting in large inward cation currents via the voltage sensor domain in response to PS stimulation. Taken together, these data indicate that the two DEE mutations in TRPM3 result in a profound gain of channel function, which may lie at the basis of epileptic activity and neurodevelopmental symptoms in the patients. 
47 Transient Receptor Potential (TRP) channel TRPM3 is a calcium-permeable cation channel that can be activated by heat (1) and by a variety of chemical ligands, including the endogenous neurosteroid pregnenolone sulphate (PS) (2). TRPM3 is expressed in a large subset of mouse and human somatosensory neurons, where it is involved in the detection of noxious heat and the development of inflammatory pain $(1,3)$. Moreover, TRPM3 is expressed in several brain areas, including the choroid plexus, cerebellum, cortex and the hippocampal formation (4-6), but its functional role in these areas is unknown. Recently, two de novo substitutions in TRPM3 (V990M and P1090Q) were identified as the cause of intellectual disability and epilepsy in eight probands with developmental and epileptic encephalopathy (DEE) (7). However, the consequences of these mutations on TRPM3 function remained elusive. We here demonstrate that both mutations lead to significant gain-of-channel function, including increased basal activity and higher sensitivity to PS and heat. V990M exhibits further pronounced functional alterations, including anomalous activation of the alternative current through the voltage-sensor domain, reduced sensitivity to receptor-mediated inhibition and calcium-dependent inactivation, and lower sensitivity to block by the anticonvulsant primidone. 


\section{Results \& Discussion}

When performing Fura-2-based calcium imaging on transiently transfected HEK293T cells, we observed significantly higher intracellular $\mathrm{Ca}^{2+}$ concentrations $\left(\left[\mathrm{Ca}^{2+}\right]_{i}\right)$ in cells expressing the two DEE mutants compared to wild type human TRPM3 (WT) (Fig. 1A,B), an effect that was much more pronounced in the V990M mutant. Application of primidone or isosakuranetin, both potent TRPM3 antagonists $(8,9)$, reduced $\left[\mathrm{Ca}^{2+}\right]_{\mathrm{i}}$ in cells expressing WT or mutant TRPM3. In absolute terms, the antagonist-induced reduction in $\left[\mathrm{Ca}^{2+}\right]_{\mathrm{i}}$ was the largest for the V990M mutant, yet $\left[\mathrm{Ca}^{2+}\right]_{\mathrm{i}}$ did not fully return to the level of cells expressing WT (Fig. 1A,C). These results suggest that the DEE mutations lead to increased basal channel activity. In line herewith, whole-cell currents in response to voltage steps revealed increased current amplitudes in cells expressing the DEE mutants compared to WT (Supplementary Fig. 1).

Next, we compared the responses of WT and DEE mutants to stimulation with agonist PS and with clotrimazole (Clt), an antifungal drug and known TRPM3 modulator (10). In line with earlier studies (2), we found that PS $(40 \mu \mathrm{M})$ reversibly activated outwardly rectifying wholecell currents in cells expressing WT, whereas application of Clt $(10 \mu \mathrm{M})$ did not activate currents by itself but potentiated responses to PS (Fig. 1D,E and Supplementary Fig. 1). In particular, application of PS in the presence of Clt provoked activation of a large inwardly rectifying current component, which has been attributed to activation of an alternative ion permeation pathway located in the voltage-sensing domain, distinct from the central pore (10). The response pattern was strikingly altered in cells expressing the DEE mutants. In the case of V990M, whole-cell currents in response to PS were significantly larger compared to WT, and, notably, exhibited a prominent inwardly rectifying current component. In addition, application of Clt induced robust currents in the absence of PS, whereas currents in the combined presence of Clt and PS were similar in amplitude and shape as WT (Fig. 1F,G and Supplementary Fig.1). In the case of P1090Q, whole-cell currents in response to PS were also significantly larger 
compared to WT, but lacked the inwardly rectifying component observed in the V990M mutant. Clt did not activate currents in cells expressing P1090Q, and, in contrast to WT, inhibited the response to PS (Fig. 1H,I and Supplementary Fig.1). Taken together, these results indicate that both DEE mutants lead to significant changes in channel gating, including increased basal activity and pronounced alterations in ligand responses.

To further assess the enhanced response to PS stimulation of the DEE mutants, we compared their apparent affinity to PS by measuring $\left[\mathrm{Ca}^{2+}\right]_{\mathrm{i}}$ responses to stepwise increases in PS concentrations. We found that the concentration-response curve for both mutants was shifted to significantly lower concentrations compared to WT. Notably, whereas a PS concentration of $10 \mu \mathrm{M}$ was required to induce a detectable response in cells expressing WT TRPM3, we observed robust calcium responses at concentrations as low as $100 \mathrm{nM}$ for $\mathrm{V} 990 \mathrm{M}$ and $1 \mu \mathrm{M}$ for P1090Q. Moreover, the maximal increase in $\left[\mathrm{Ca}^{2+}\right]_{\mathrm{i}}$ to saturating PS concentrations was significantly higher in P1090Q expressing cells compared to WT (Fig. 2A,B). Thus, both DEE mutants show increased responses to the neurosteroid PS.

TRPM3 is a temperature-sensitive channel, activated upon heating (1). To address whether the DEE mutations affect the channel's response to heat, we compared changes in $\left[\mathrm{Ca}^{2+}\right]_{\mathrm{i}}$ in cells expressing WT or mutant TRPM3 upon exposure to a heat ramp from 23 to $40{ }^{\circ} \mathrm{C}$. Compared to non-transfected cells, we measured a significantly larger increase in $\left[\mathrm{Ca}^{2+}\right]_{\mathrm{i}}$ in cells expressing WT or mutant TRPM3. Notably, the amplitude of the heat-induced response was significantly larger in cells expressing the DEE mutants compared to WT (Fig. 2C,D).

TRPM3 activity is inhibited upon activation of $\mathrm{G}$ protein-coupled receptors, via a mechanism that involves direct binding of the $\mathrm{G}_{\beta \gamma}$ of trimeric G-proteins to the channel (11-13). To evaluate whether $\mathrm{G}_{\beta \gamma}$-dependent modulation is altered in the DEE mutations, we co-transfected HEK293T cells with the $\mu$-opioid receptor and WT or mutant TRPM3, and evaluated the effect of the selective agonist DAMGO on PS-activated whole-cell currents. In cells expressing WT 
or P1090Q, application of $1 \mu \mathrm{M}$ DAMGO induced a complete and rapidly reversible inhibition of inward and outward currents, whereas V990M was only partly inhibited (Fig. 2E-I). The DAMGO concentration for half-maximal inhibition of the PS-activated currents shifted from $4.0 \pm 0.6 \mathrm{nM}$ for WT to $40 \pm 10 \mathrm{nM}$ for V990M (Supplementary Fig.2). Note that DAMGO was without effect on TRPM3 currents in cells that were not co-transfected with the $\mu$-opioid receptor (Supplementary Fig.2). A difference in sensitivity was also found for the anticonvulsant drug primidone, which at a concentration of $25 \mu \mathrm{M}$ caused a full inhibition of PS-activated inward and outward currents mediated by WT or P1090Q, but blocked currents mediated by V990M by only 50\% (Fig. 2E-G,I). A similar difference in primidone sensitivity was observed using Fura-2-based calcium imaging (Supplementary Fig.2).

When switching from the standard, $\mathrm{Ca}^{2+}$-free extracellular solution to a solution containing 1 mM Ca ${ }^{2+}$, PS-activated currents mediated by WT or by P1090Q undergo time-dependent desensitization $(10,14)$. In contrast, PS-activated currents mediated by V990M remained stable in the presence of extracellular $\mathrm{Ca}^{2+}$, indicating reduced sensitivity to $\mathrm{Ca}^{2+}$-dependent desensitization in this mutant (Fig. 2J-M).

The PS-induced whole-cell currents mediated by V990M showed a prominent inwardly rectifying current component. A similar inwardly rectifying current component can also be activated in WT TRPM3 when PS is applied in the presence of Clt. In earlier work, we have demonstrated that this inwardly rectifying current component represents ion flux through an alternative ion permeation pathway located in the voltage sensor domain of TRPM3, which can be distinguished from the central pore based on its voltage dependence, insensitivity to pore block by $\mathrm{La}^{3+}$ and ion selectivity (including a lower permeability for monomethylammonium $\left(\mathrm{MMA}^{+}\right)$compared to $\left.\mathrm{Na}^{+}\right)(10,14)$. Notably, the V990M mutation is located in close vicinity of Asp988 and Gly991, which we recently identified as critical determinants of the alternative ion permeation pathway (15). We therefore hypothesized that gating of the alternative ion 
permeation pathway is facilitated in V990M, such that it can be activated by PS even when Clt is not co-applied. We found that the PS-activated current in the V990M mutant showed a bimodal voltage dependence (Fig. 3B), its inward current component was resistant to block by the central pore blocker $\mathrm{La}^{3+}$ (Fig. 3F,G), and inward currents were reduced when extracellular $\mathrm{Na}^{+}$was replaced by $\mathrm{MMA}^{+}$(Fig. 3H). Taken together, these data indicate that the V990M mutation leads to a gain of function at the level of the alternative ion permeation pathway.

Considering that all reported DEE patients were heterozygous for the TRPM3 substitutions (7), and that TRPM3 is functional as a tetramer, it is expected that patients express heteromultimeric channels consisting of variable numbers of WT and mutant subunits. To mimic this situation in vitro, we performed a limited number of experiments in cells co-transfected with a mixture of cDNA encoding WT and mutant TRPM3 in a 1:1 ratio. The amplitude of PS-induced inward currents in cells expressing a WT:V990M mixture was intermediate between cells expressing only WT or only V990M (Fig. 4A,B). Moreover, the PS-activated currents exhibited the typical inwardly rectifying current component (Fig. 4C,D). Finally, in contrast to WT but like V990M, Clt $(10 \mu \mathrm{M})$ activated robust currents in cells expressing a WT:V990M mixture (Fig. 4A,B). rectification pattern of the PS-induced currents that was different form the homozygote situation (Fig. 4G). Moreover, the effect of Clt pre-incubation was different in co-transfected cells. WT:P1090Q cells showed first a potentiation of the PS responses by pre-incubation of Clt, followed by a time-dependent current inhibition. This was in contrast to the block of PS-induced currents by pre-incubation with Clt for the P1090Q mutant in isolation (Fig. 4H). In conclusion, our results indicate that two human mutations in the TRPM3 gene associated with DEE give rise to channels with substantially altered functional properties. Whereas the V990M and P1090Q mutations have various differential effects on several aspects of TRPM3 
162 gating, both can be considered as strong gain-of-function mutants, with increased inward cation 163 currents and $\mathrm{Ca}^{2+}$ influx under basal condition or when stimulated with heat or the endogenous 164 neurosteroid PS. We hypothesize that the increased calcium influx and depolarizing channel 165 activity may lie at the basis of seizure development and neurodevelopmental symptoms in DEE 166 patients. 
168 We thank all the members of the Laboratory of Ion Channel Research and the Laboratory of 169 Endometrium, Endometriosis and Reproductive Medicine at the KU Leuven, for their helpful 170 discussions and comments.

\section{Funding}

172 This project has received funding from the Belgian Federal Government (IUAP P7/13 to T.V.), 173 the Research Foundation-Flanders (G.0565.07, G.0825.11 to T.V. and J.V. G.084515N and 174 G.0B1819N to J.V.), the Research Council of the KU Leuven (C1-TRPLe to T.V.), the Queen 175 Elisabeth Medical Foundation for Neurosciences (to T.V.), the Belgian Foundation Against 176 Cancer (to J.V. and T.V.) K.H. is a 'Postdoctoral Fellow' of the Research Foundation 177 Flanders, Belgium

\section{Competing interest}

179 The authors declare no conflict of interest. 


\section{References}

1. Vriens J, Owsianik G, Hofmann T, Philipp SE, Stab J, Chen X, et al. TRPM3 is a nociceptor channel involved in the detection of noxious heat. Neuron. 2011;70(3):482-94.

2. Wagner TF, Loch S, Lambert S, Straub I, Mannebach S, Mathar I, et al. Transient receptor potential M3 channels are ionotropic steroid receptors in pancreatic beta cells. Nat Cell Biol. 2008;10(12):1421-30.

3. Vangeel L, Benoit M, Miron Y, Miller PE, De Clercq K, Chaltin P, et al. Functional expression and pharmacological modulation of TRPM3 in human sensory neurons. Br J Pharmacol. 2020.

4. Grimm C, Kraft R, Sauerbruch S, Schultz G, Harteneck C. Molecular and functional characterization of the melastatin-related cation channel TRPM3. J Biol Chem. 2003;278(24):21493501.

5. Oberwinkler J, Lis A, Giehl KM, Flockerzi V, Philipp SE. Alternative splicing switches the divalent cation selectivity of TRPM3 channels. J Biol Chem. 2005;280(23):22540-8.

6. Zamudio-Bulcock PA, Everett J, Harteneck C, Valenzuela CF. Activation of steroid-sensitive TRPM3 channels potentiates glutamatergic transmission at cerebellar Purkinje neurons from developing rats. J Neurochem. 2011;119(3):474-85.

7. Dyment DA, Terhal PA, Rustad CF, Tveten K, Griffith C, Jayakar P, et al. De novo substitutions of TRPM3 cause intellectual disability and epilepsy. Eur J Hum Genet. 2019;27(10):1611-8.

8. Krugel U, Straub I, Beckmann H, Schaefer M. Primidone inhibits TRPM3 and attenuates thermal nociception in vivo. Pain. 2017;158(5):856-67.

9. Straub I, Krugel U, Mohr F, Teichert J, Rizun O, Konrad M, et al. Flavanones that selectively inhibit TRPM3 attenuate thermal nociception in vivo. Mol Pharmacol. 2013;84(5):736-50.

10. Vriens J, Held K, Janssens A, Toth BI, Kerselaers S, Nilius B, et al. Opening of an alternative ion permeation pathway in a nociceptor TRP channel. Nat Chem Biol. 2014;10(3):188-95.

11. Badheka D, Yudin Y, Borbiro I, Hartle CM, Yazici A, Mirshahi T, et al. Inhibition of Transient Receptor Potential Melastatin 3 ion channels by G-protein betagamma subunits. Elife. 2017;6.

12. Dembla S, Behrendt M, Mohr F, Goecke C, Sondermann J, Schneider FM, et al. Anti-nociceptive action of peripheral mu-opioid receptors by G-beta-gamma protein-mediated inhibition of TRPM3 channels. Elife. 2017;6.

13. Quallo T, Alkhatib O, Gentry C, Andersson DA, Bevan S. G protein betagamma subunits inhibit TRPM3 ion channels in sensory neurons. Elife. 2017;6.

14. Held K, Kichko T, De Clercq K, Klaassen H, Van Bree R, Vanherck JC, et al. Activation of TRPM3 by a potent synthetic ligand reveals a role in peptide release. Proc Natl Acad Sci U S A. 2015;112(11):E1363-72.

15. Held K, Gruss F, Aloi VD, Janssens A, Ulens C, Voets T, et al. Mutations in the voltage-sensing domain affect the alternative ion permeation pathway in the TRPM3 channel. J Physiol. 2018;596(12):2413-32. 


\section{Figure legends}

Figure 1: Elevated basal activity in HEK293T cells expressing TRPM3 DEE mutants

(A) Time course of intracellular calcium concentrations $\left(\left[\mathrm{Ca}^{2+}\right]_{i}\right)( \pm$ SEM) upon application of the TRPM3 inhibitors primidone $(100 \mu \mathrm{M})$ and isosakuranetin $(50 \mu \mathrm{M})$ for $\mathrm{WT}(\mathrm{n}=230)$, P1090Q $(n=163)$ and V990M $(n=79)$ transfected HEK293T cells, and non-transfected (NT) cells $(\mathrm{n}=93)(\mathrm{N}=3$ independent experiments). (B) Basal intracellular calcium concentrations represented as mean \pm SEM. (C) Primidone-induced decrease in $\left[\mathrm{Ca}^{2+}\right]_{\mathrm{i}}$ for the indicated cells. Data are represented as mean \pm SEM. $(\mathbf{D}, \mathbf{F}$ and $\mathbf{H})$ Amplitude of currents at a holding potential of $+80 \mathrm{mV}$ and $-80 \mathrm{mV}$ (measured with voltage ramps) upon application of PS (40 $\mu \mathrm{M})$, Clt $(10 \mu \mathrm{M})$ and co-application of PS and Clt for WT $(\mathrm{n}=10)(\mathbf{D}), \mathrm{V} 990 \mathrm{M}(\mathrm{n}=7)(\mathbf{F})$ and P1090Q $(\mathrm{n}=9)(\mathbf{H})$. (E, $\mathbf{G}$ and I) Current-voltage relationships at the time points indicated in $(\mathbf{D}),(\mathbf{F})$ and $(\mathbf{G}) .{ }^{* * *} \mathrm{p}<0.001$ (Kruskal-Wallis ANOVA with Dunn's posthoc test).

Figure 2: Altered sensitivity of DEE mutants for thermal stimulation and pharmacological modulation

(A) Time course of $\left[\mathrm{Ca}^{2+}\right]_{\mathrm{i}}( \pm \mathrm{SEM})$ upon application of the TRPM3 agonist PS in stepwise increasing dose $(0.01-100 \mu M)$ for WT $(\mathrm{n}=615)$, V990M $(\mathrm{n}=130)$ and P1090Q $(\mathrm{n}=196)$ ( $\mathrm{N}=3$ independent experiments). (B) PS concentration-response curves for $\mathrm{WT}\left(\mathrm{EC}_{50}=14.3\right.$ $\pm 5.8 \mu \mathrm{M}), \mathrm{V} 990 \mathrm{M}\left(\mathrm{EC}_{50}=2.1 \pm 0.4 \mu \mathrm{M}\right)$, and P1090Q $\left(\mathrm{EC}_{50}=7.5 \pm 1.4 \mu \mathrm{M}\right) .(\mathrm{C})$ Time course of $\left[\mathrm{Ca}^{2+}\right]_{\mathrm{i}}$ for NT (gray, $\mathrm{n}=46$ ), WT (black, $\mathrm{n}=148$ ), V990M (blue, $\mathrm{n}=259$ ) and P1090Q (red, $\mathrm{n}=271$ ) when applying a heat ramp (magenta). Analysis of 3 independent experiments, where the data are represented as mean \pm SEM. (D) Corresponding amplitudes of the temperature response, represented as mean \pm SEM. (E-G) Amplitude of currents at $+80 \mathrm{mV}$ and $-80 \mathrm{mV}$ (measured during voltage ramps) upon application of PS $(40 \mu \mathrm{M})$ with co-application of the $\mu$ opioid receptor agonist DAMGO $(1 \mu \mathrm{M})$ or the TRPM3 inhibitor primidone $(25 \mu \mathrm{M})$ for WT $(\mathrm{n}=6)(\mathbf{E})$, V990M $(\mathrm{n}=8)(\mathbf{F})$ and P1090Q $(\mathrm{n}=6)(\mathbf{G})$, in cells co-expressing the $\mu$-opioid 
receptor. (H-I) Percentage inhibition of PS-induced currents upon application of DAMGO (H) and primidone (I) for WT (black), V990M (blue) and P1090Q (red). The filled and shaded bars represent the current inhibition at $-80 \mathrm{mV}$ and $+80 \mathrm{mV}$, respectively. (J-L) Amplitude of currents at $+80 \mathrm{mV}$ and $-80 \mathrm{mV}$ (measured during voltage ramps) upon application of PS (40 $\mu \mathrm{M})$ in the presence of $1 \mathrm{mM}$ extracellular calcium for WT $(\mathrm{n}=8)(\mathbf{J})$, V990M $(\mathrm{n}=6)(\mathbf{K})$ and P1090Q (n= 6) (L). (M) Percentage inhibition upon calcium application for WT, V990M (VM) and P1090Q (PQ) (mean \pm SEM). $* * \mathrm{p}<0.01$ and $* * * \mathrm{p}<0.001$ (Kruskal-Wallis ANOVA with Dunn's posthoc test).

\section{Figure 3: Altered gating of the alternative pore in V990M}

(A-C) $G$ - $V$ plots of PS-activated currents for (A) WT (black), (B) V990M (blue) and (C) P1090Q (red). Currents measured during voltage-steps ranging from $-200 \mathrm{mV}$ to $+200 \mathrm{mV}$, separated by steps of $+50 \mathrm{mV}$. Representative currents are shown as insets in each graph; $\mathrm{n}=6$ for each experiment. (D) Rectification pattern of PS (40 $\mu \mathrm{M})$ (full circle and line) and PS + Clt (10 $\mu \mathrm{M}$ )-induced (open circle and dashed line) currents for WT, V990M and P1090Q. Data points are derived by plotting the current increase at $+150 \mathrm{mV}$ versus the current increases at $-150 \mathrm{mV} ; \mathrm{n} \geq 4$ for each dataset. (E) Time course of WT TRPM3 whole-cell currents at \pm 150 $\mathrm{mV}$ upon application of PS $(40 \mu \mathrm{M})$ and Lanthanum $\left(\mathrm{La}^{3+} ; 10 \mu \mathrm{M}\right)$ or PS + Clt and $\mathrm{La}^{3+} .(\mathbf{F})$ Time course of V990M mutant whole-cell currents at $\pm 150 \mathrm{mV}$ upon application of PS and $\mathrm{La}^{3+}$. (G) Relative $\mathrm{La}^{3+}$ block calculated from experiments as in E) and F) for WT (black) in presence PS + Clt $(n=4)$ and for V990M (blue, $n=8)$ in presence of PS alone. $(\mathbf{H})$ Relative PS-induced currents at $-150 \mathrm{mV}$ carried by monomethylammonium $\left(\mathrm{MMA}^{+}\right)$in WT (black), V990M (blue) and P1090Q (red). $\mathrm{MMA}^{+}$currents were normalized to the currents carried by $\mathrm{Na}^{+}$; PS $(40 \mu \mathrm{M})$ and $\mathrm{n}=5$ for all experiments. $* * \mathrm{p}<0.01$ (Kruskal-Wallis ANOVA with Dunn's posthoc test).

\section{Figure 4: Heterozygous effects of DEE mutants}


(A) Time course of whole-cell currents at $\pm 80 \mathrm{mV}$ recorded in HEK293T cells transiently cotransfected with WT and V990M mutant DNA (1:1) upon application of PS (40 $\mu \mathrm{M})$, Clt (10 $\mu \mathrm{M}$ ) or PS + Clt. (B) Current amplitudes at a holding potential of $-80 \mathrm{mV}$ (measured with voltage ramps) upon application of PS $(40 \mu \mathrm{M})$ for WT $(\mathrm{n}=10)$ (black), VM $(\mathrm{n}=7)$ (light blue) 272 or WT + VM (1:1) $(\mathrm{n}=7)$ (dark blue). (C) Same as in (B) but for current amplitude ratios of $273+80 \mathrm{mV} /-80 \mathrm{mV}$. (D) $G$ - $V$ plots for PS-activated WT TRPM3 (black), V990M (light blue) and WT+V990M (1:1) (dark blue). Data points were obtained with a step protocol ranging from $200 \mathrm{mV}$ to $+200 \mathrm{mV}$ with $+50 \mathrm{mV}$ steps; $\mathrm{n} \geq 6$ for each experiment. (E) Time course of wholecell currents at $\pm 80 \mathrm{mV}$ recorded in HEK293T cells transiently co-transfected with WT and P1090Q (1:1) upon application of PS $(40 \mu \mathrm{M})$, Clt $(10 \mu \mathrm{M})$ or PS + Clt. (F) Similar as in (B) 278 but for WT $(\mathrm{n}=10)$ (black), P1090Q $(\mathrm{n}=9)$ (light red) or co-transfected WT + P1090Q (1:1) $279(\mathrm{n}=5)($ dark red). (G) Similar as in $(\mathrm{C})$ but for WT $(\mathrm{n}=10)$ (black), P1090Q $(\mathrm{n}=9)$ (light red) or co-transfected WT + P1090Q (1:1) (dark red). (H) X-fold potentiation at peak and steadystate conditions of Clt-potentiated PS-currents in WT (black), P1090Q (light red) or cotransfected with WT + P1090Q (1:1) (dark red); $\mathrm{n} \geq 4 . * \mathrm{p}<0.05, * * \mathrm{p}<0.01$ and $* * * \mathrm{p}<$ 0.001 (Kruskal-Wallis ANOVA with Dunn's posthoc test for panel B, F and H. One-way ANOVA with Tukey's posthoc test for panel C and G). 
Figure 1

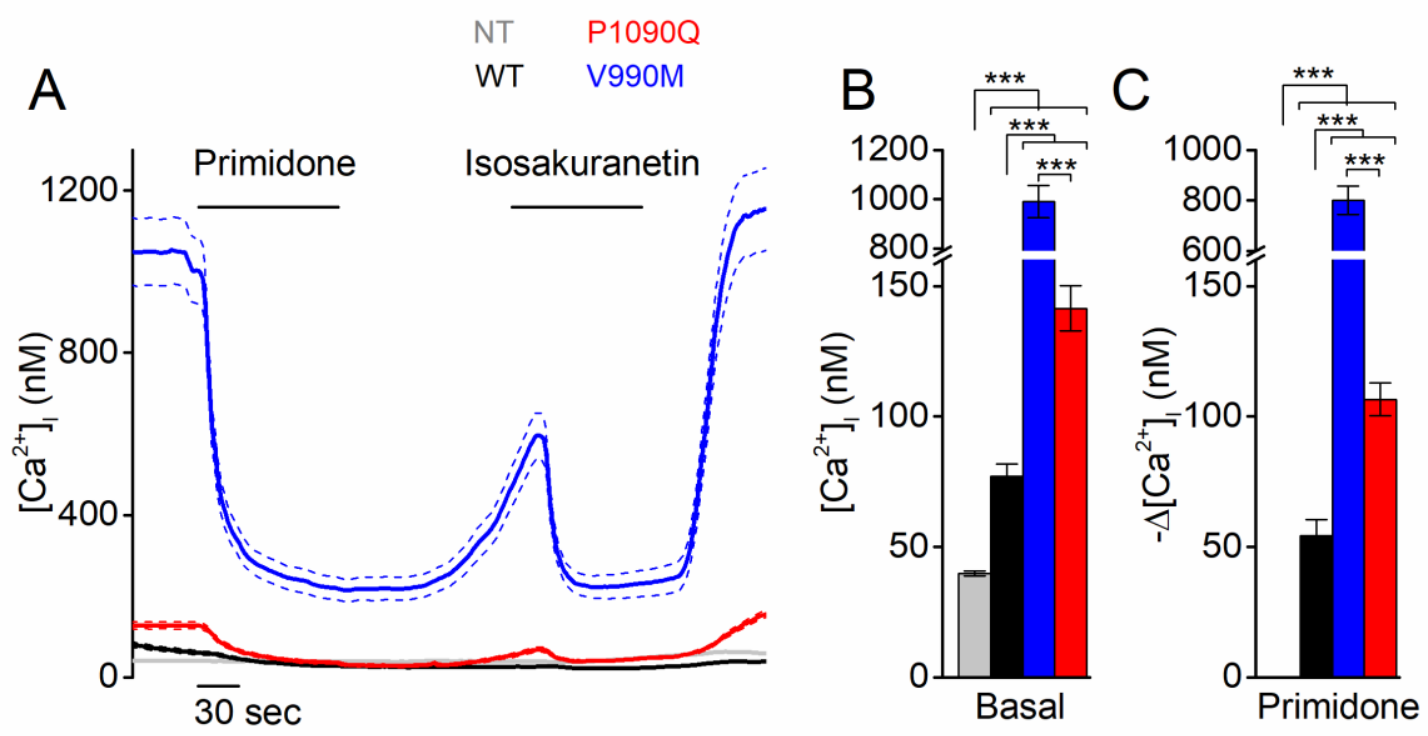

D

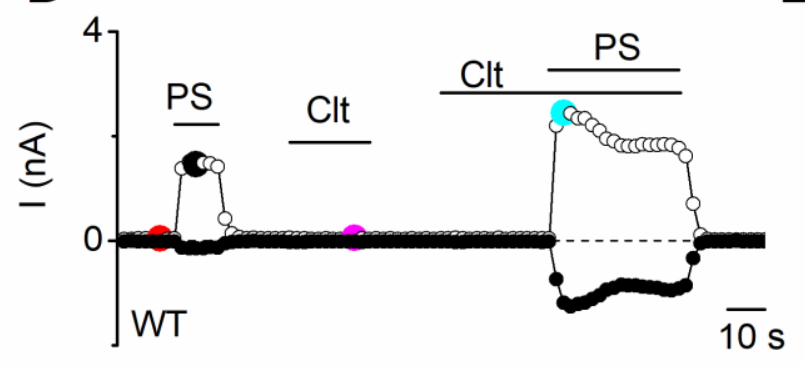

$\mathrm{F}$

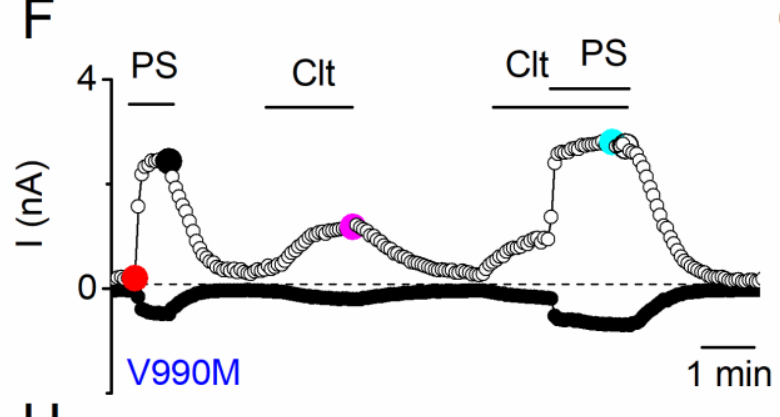

$\mathrm{H}$

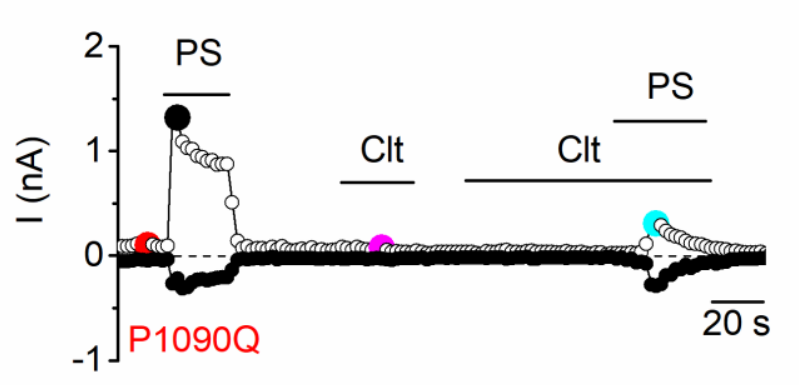

E
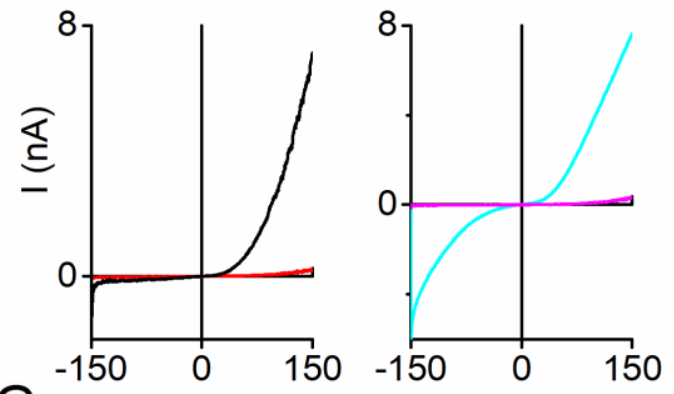

G
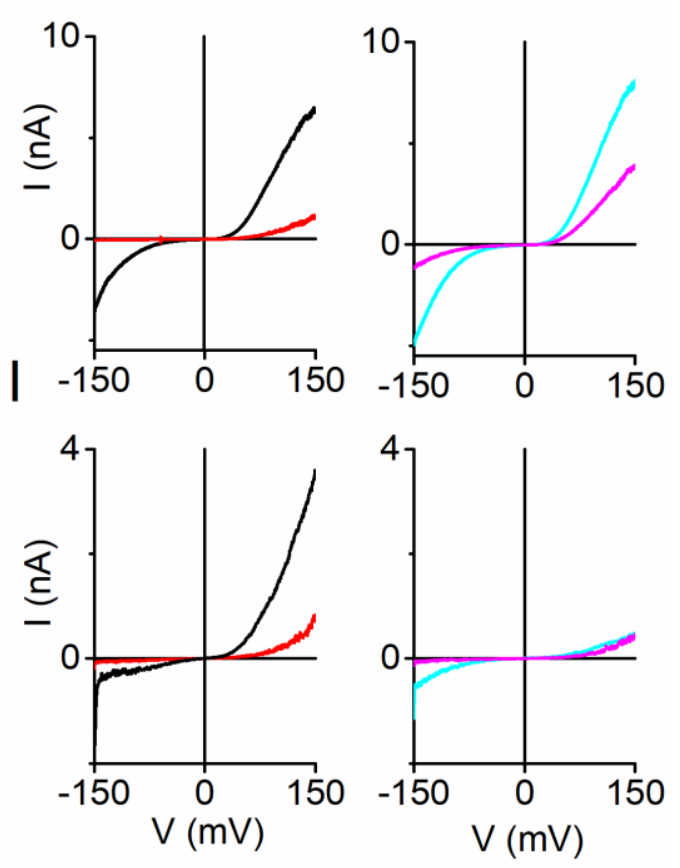
Figure 2

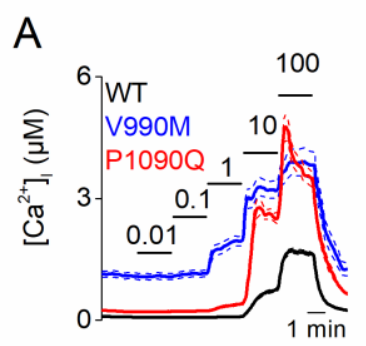

E
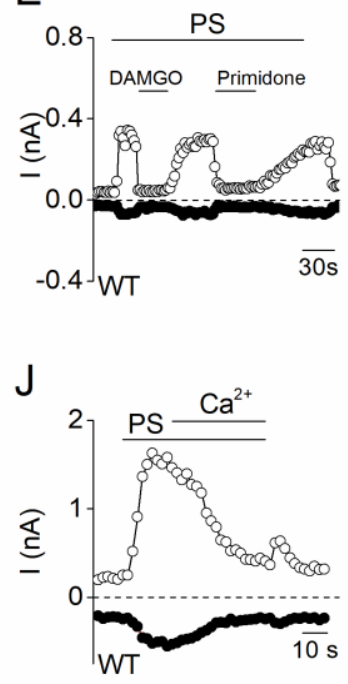

B

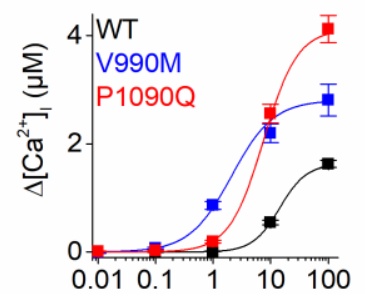

F

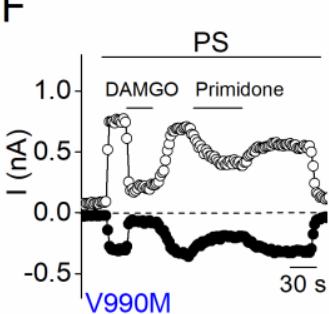

K

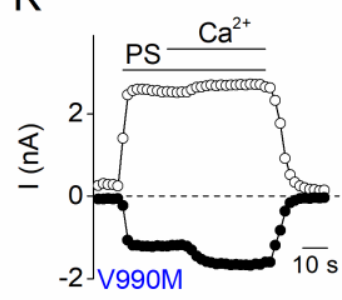

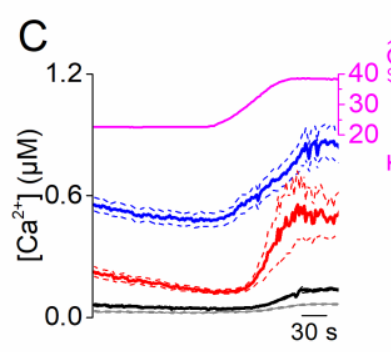

G

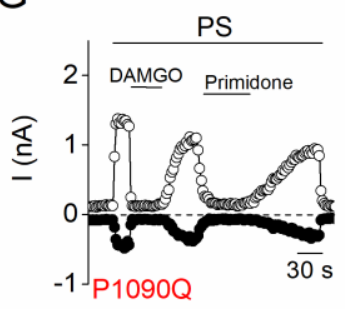

L

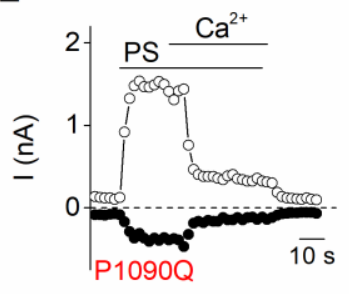

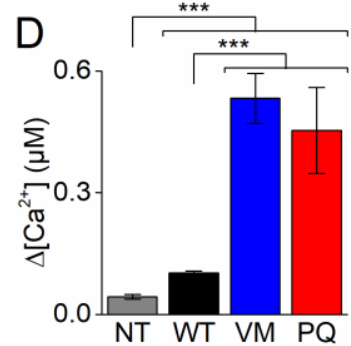

$\mathrm{H}$

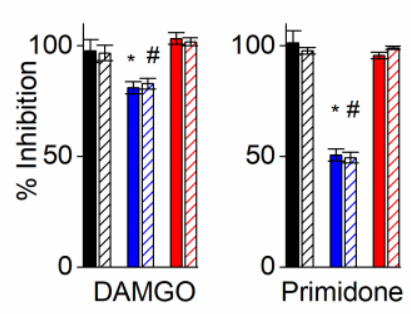

M

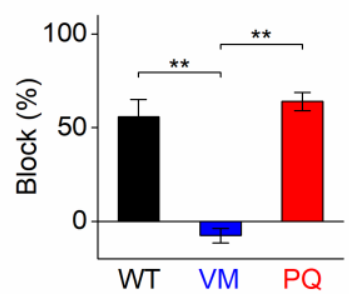


Figure 3
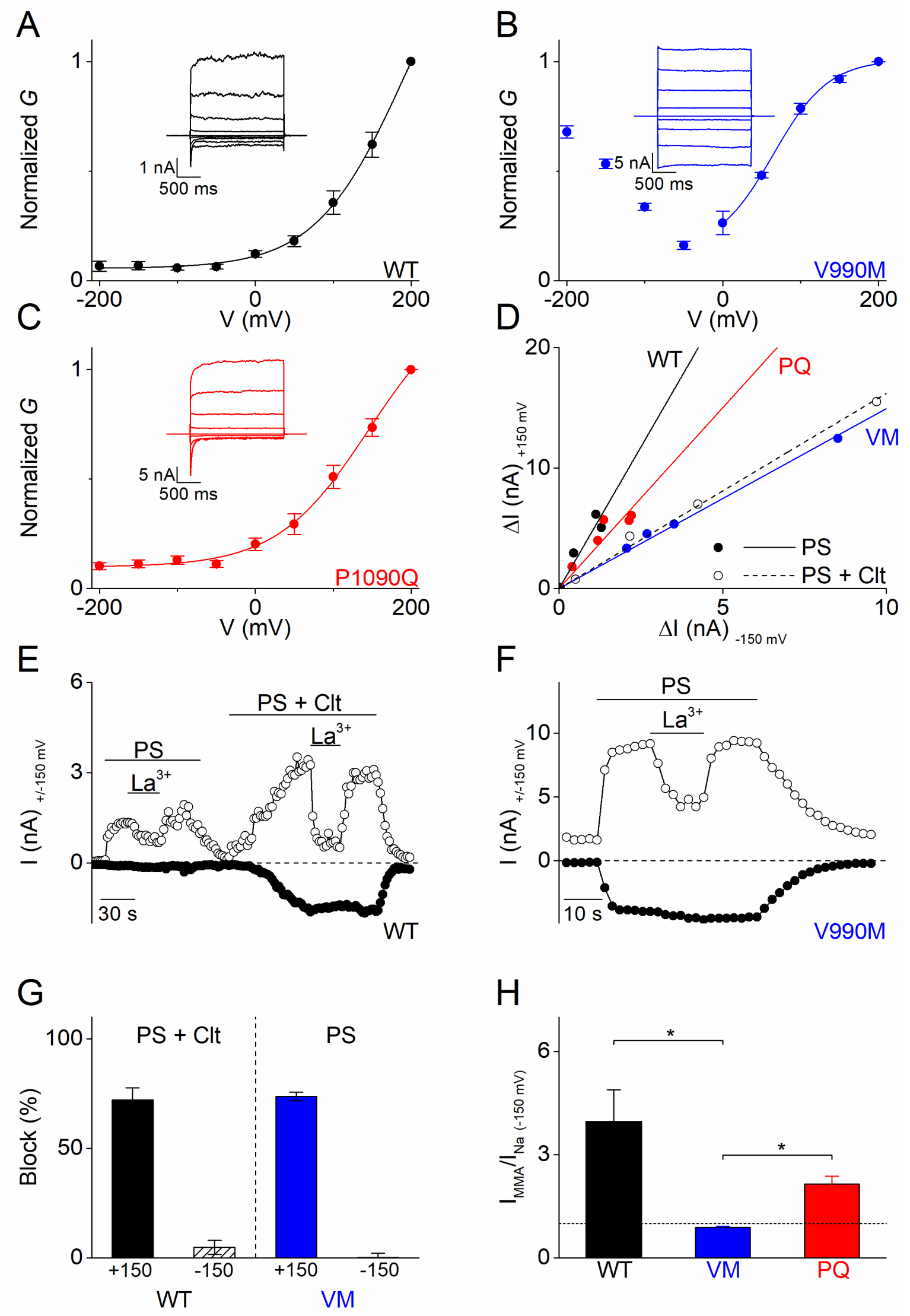

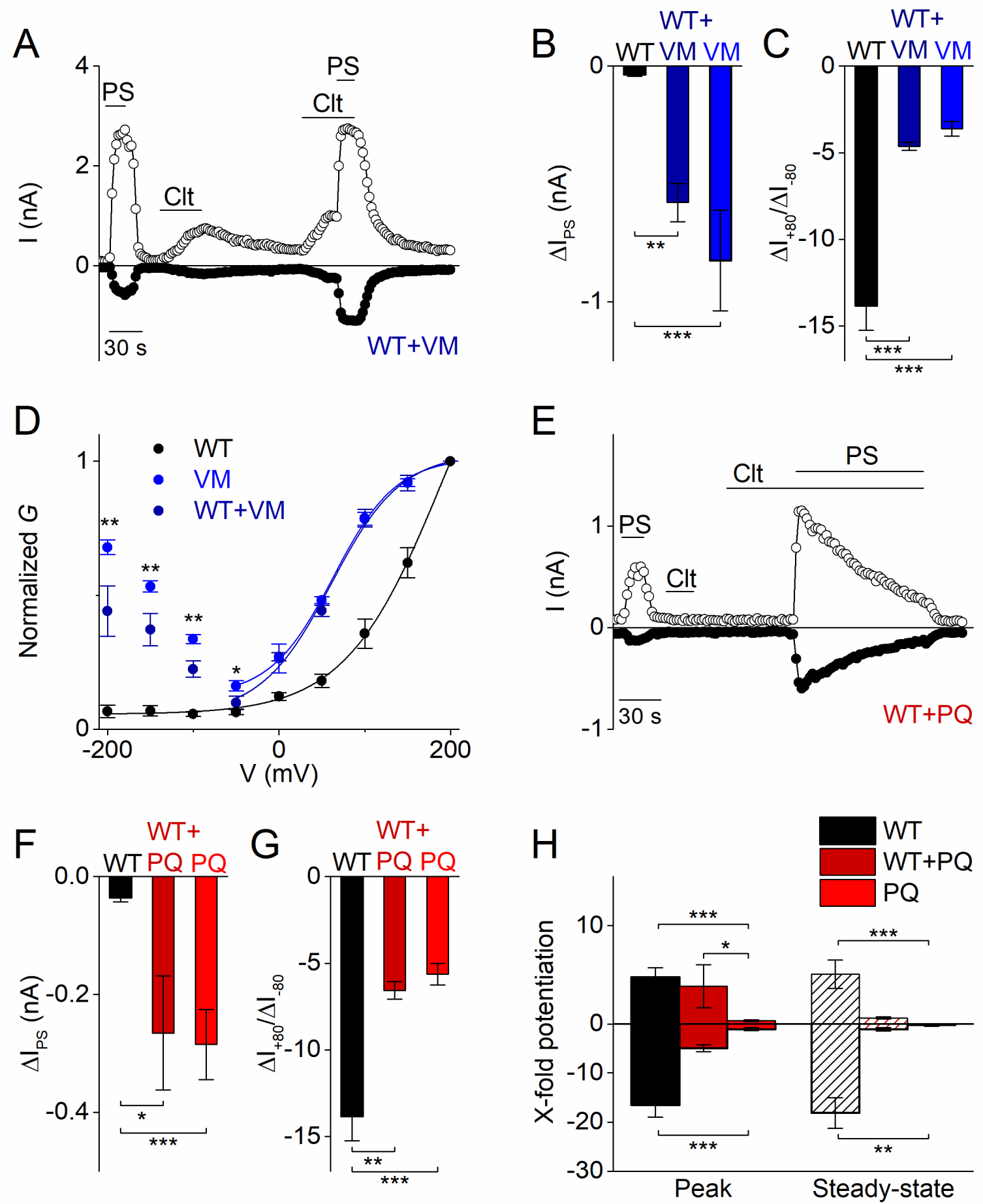